\title{
Parallel Changes in Positive Youth Development and Self-awareness: the Role of Emotional Self-regulation, Self-esteem, and Self-reflection
}

\author{
Esther C. A. Mertens ${ }^{1}\left[\right.$ [D $\cdot$ Maja Deković ${ }^{1} \cdot$ Monique van Londen $^{1} \cdot$ Ellen Reitz $^{1}$
}

Accepted: 14 January 2022 / Published online: 28 January 2022

(c) The Author(s) 2022

\begin{abstract}
The aim of the present study was to examine whether early adolescents' positive development (i.e., resilience and psychological wellbeing) could be enhanced by stimulating three dimensions of self-awareness: emotional self-regulation, selfesteem, and self-reflection. An experimental field study (randomized controlled trial) was conducted. Seventh grade students $\left(N=1299 ; M_{\text {age }}=12.38 ; 54 \%\right.$ boys $)$ completed multiple assessments at the beginning, during, and immediately after the intervention or at parallel time points for students in the control condition. Changes in the assessed constructs were examined with parallel process latent growth curve models. The results showed that increases in emotional self-regulation and self-esteem were related to concurrent increases in both resilience and psychological wellbeing. Changes in self-reflection were, however, not related to changes in these outcomes. The trajectories of change and parallel processes were similar in the intervention and control condition. These findings suggest that interventions aiming to stimulate positive youth development might be optimized by also focusing on youth's self-awareness, though more knowledge about how self-awareness can be stimulated best is needed. This trial was registered in the Dutch Trial Register, number NL6371 (old number: NTR6554), on July 3, 2017.
\end{abstract}

Keywords Self-awareness $\cdot$ Emotional self-regulation $\cdot$ Self-esteem $\cdot$ Self-reflection $\cdot$ Parallel change $\cdot$ School-based intervention $\cdot$ Positive youth development

\section{Introduction}

Studying how positive youth development can be stimulated is pivotal since positive outcomes can provide foundations for healthy development as well as function as protective factors for decreasing problem behaviors (Greenberg et al., 2017). Especially youth's resilience (i.e., the ability to adapt to change and stressful events in a healthy and flexible way; Catalano et al., 2004) and psychological wellbeing (i.e., the presence of positive feelings and absence of negative feelings; Ravens-Sieberer \& The European KIDSCREEN Group, 2006) appear important positive outcomes to strengthen (Benson et al., 2007; Catalano et al., 2004). Self-awareness has been indicated by the Collaborative for Academic, Social, and Emotional Learning (CASEL) as an important competence that provides a foundation for

Esther C. A. Mertens

e.c.a.mertens@uu.nl

1 Department of Clinical Child and Family Studies, Utrecht University, Heidelberglaan 1, 3584 CS Utrecht, The Netherlands enhanced positive youth development. Therefore, the aim of the present study is to examine parallel changes in selfawareness and both youth's resilience and psychological wellbeing. Unraveling associations between changes in one variable and changes in another variable provides not only insights in one's development, but can also contribute to understanding individual differences (Cheong et al., 2003). This knowledge can subsequently inform theory and optimization of interventions (O'Rourke \& MacKinnon, 2018).

\section{Self-awareness and Positive Development}

Self-awareness is a multidimensional overarching theoretical concept that can be defined as "understanding your own emotions, values, and personal goals" (Greenberg et al., 2017, p. 14). It concerns the tendency to focus and reflect on one's inner processes and own experiences, as well as being aware of other's perceptions (Sutton, 2016). A high level of self-awareness is characterized by recognition of one's own thoughts, feelings, and actions, as well as feeling accepted by others (Greenberg et al., 2017; Sutton, 2016). Based on 
this conceptualization of self-awareness, the competencies emotional self-regulation, self-esteem, and self-reflection seem to be key dimensions of self-awareness (Sutton, 2016).

According to CASEL's conceptual framework (Greenberg et al., 2017), improving self-awareness can, in turn, improve one's positive development. This proposed link is supported by empirical research. For instance, Morrish et al. (2018) concluded in their review that research has linked adaptive emotional self-regulation to more resilience and better psychological wellbeing. Also, higher self-esteem has been associated with better adjustment, such as mental health and happiness, and can buffer the impact of stressful events (see for a review Mann et al., 2004). Research relating selfreflection to positive development is, however, inconclusive. For instance, Elliot and Coker (2008) found in a community sample that more self-reflection was related to more happiness, whereas, in a comparable community sample, Lyke (2009) did not find a relation between self-reflection and happiness nor between self-reflection and life satisfaction. Thus, all three key dimensions of self-awareness have been linked to positive youth development, though the exact role of self-reflection remains somewhat ambiguous.

\section{An Intervention Context}

An intervention that aims to enhance youth's positive development by stimulating the three key dimensions of selfawareness, i.e., their emotional self-regulation, self-esteem, and self-reflection, is Rock and Water (R\&W; Ykema, 2002, 2018). R\&W aims to improve students' emotional self-regulation, self-esteem, and self-reflection by using a combination of a physical and psychological approach (i.e., a psychophysical approach). For instance, the intervention theorizes that emotional self-regulation is stimulated by making students aware of physical representations of their emotions, such as muscle tension and high breathing. Students are taught how to actively relax their muscles and lower their breathing to become calm. Self-esteem is addressed explicitly by encouraging an upright body posture and implicitly by creating experiences of success during the lessons. Self-reflection is stimulated by a moment of reflection after each exercise guided by questions of the $\mathrm{R} \& \mathrm{~W}$ trainer (Ykema, 2002, 2018).

The intervention is based on the theory of the " $\mathrm{R} \& \mathrm{~W}$ house" (Ykema, 2002, 2018). The competencies emotional self-regulation, self-esteem, and self-reflection are the pillars of the house. Developing these basic competencies is theorized to enable students to develop themselves on broader competencies such as resilience and psychological wellbeing. Small-scaled evaluation studies using single group pretest-posttest design showed improvements in participants' resilience, positive identity, and coping styles (Ykema et al., 2006). Additionally, a recent large-scaled randomized controlled trial (RCT) showed improvements in participants' coping strategies, self-regulation, and self-efficacy (De Graaf et al., 2016). However, the role of the three dimensions of self-awareness, the pillars of the R\&W house, in these intervention effects remains unclear.

\section{The Current Study}

In the present study, we empirically tested the CASEL's framework by examining whether changes in self-awareness (i.e., emotional self-regulation, self-esteem, and self-reflection) were related to concurrent changes in youth's positive development (i.e., resilience and psychological wellbeing). In addition, we examined in an experimental field study whether these parallel changes could be affected by an intervention, R\&W (Ykema, 2002, 2018). Experimental manipulation empowers the argument that changes in one construct could be responsible for changes in the other construct (Kazdin, 2007). The intervention was implemented for 7 th grade students following the prevocational education track (i.e., the lowest level of three educational tracks in the Dutch secondary school system). In general, this group of students has an increased risk of developing behavioral and peer related problems (Stevens \& De Looze, 2018), making it important to gain more insight in the way their positive development is shaped. We hypothesized that changes in adolescents' emotional selfregulation, self-esteem, and self-reflection would be related to concurrent changes in their resilience and psychological wellbeing. In addition, we hypothesized that adolescents in the $\mathrm{R} \& \mathrm{~W}$ condition would show a stronger increase in emotional self-regulation, self-esteem, and self-reflection which would be related to a stronger concurrent increase in resilience and psychological wellbeing, compared to adolescents receiving care as usual (i.e., control condition).

Our study adds to the literature in four ways. First, even though self-awareness is a multidimensional concept (Greenberg et al., 2017), often only one dimension of self-awareness is assessed (e.g., Weytens et al., 2014). It is important to examine multiple dimensions of self-awareness as these might relate differently to positive youth development. Second, despite the focus of CASEL's conceptual framework on youth's positive development, studies predominantly focus on maladjustment and problem behaviors (e.g., Blossom et al., 2020), rather than on adjustment and positive outcomes, especially when youth have an increased risk of developing problems. In order to gain insights in how the positive development of these at risk youth can be stimulated, it is eminent to also focus on positive outcomes (Benson et al., 2007). Third, although metacognitions such as self-awareness develop particularly during 
early adolescence, as adolescents' cognitive and emotional capacities increase (Barber, 2005), only few studies explicitly focus on (early) adolescents. Instead, studies focus on children (e.g., Rimm-Kaufman \& Hulleman, 2014), both children and adolescents (e.g., Morrish et al., 2018), or older youth (e.g., Bakker \& Rickard, 2018). Fourth, previous longitudinal research has examined (dimensions of) self-awareness as predictor of later positive outcomes. However, the developmental trajectories of self-awareness and positive outcomes may be related. By examining parallel change, potential mechanisms that shape development may be uncovered (Cheong et al., 2003).

\section{Method}

\section{Design and Procedure}

Data for the current study were collected as part of a larger project examining the effectiveness of R\&W by means of a 2-year RCT (see Mertens et al., 2018 for the study protocol). This RCT consisted of three intervention conditions that differed in the number of people involved in the intervention (i.e., light: a core team of teachers; standard: all teachers; plus: all teachers and parents) and a control condition (i.e., care as usual).

Schools were eligible for inclusion if they offered the prevocational education track and had not implemented $\mathrm{R} \& \mathrm{~W}$ in the last 2 years. Using an online random number generator, the first author randomly allocated 13 schools in urban and rural areas throughout the Netherlands to the conditions (1:1:1:1). To enhance an equal distribution of students over the conditions, schools were stratified by school size (small to moderate sized schools with $<100$ students in the 7th grade, large schools with $>100$ students in the 7th grade) in blocks of four (i.e., the number of conditions). After randomization but before the start of data collection, one school in the control condition dropped out due to a change in school management and was replaced by another school (see Fig. 1). As the focus in the present study is on parallel change rather than its effectiveness, we merged the data of the three intervention conditions to form one intervention condition. Additionally, we only used data of the first year of the RCT as intervention effects were mostly established during the first part of intervention (see Mertens et al., 2021).

Students completed questionnaires before the intervention started, at baseline (T0; October/November 2017), after each third intervention lesson of the 14 lessons (4 measurement points), and after completing the first year of the intervention (T5; March/April 2018). The interim measurements were shortened questionnaires based on face validity and factor loadings of the items in previous studies. Students gave active informed consent for completing the questionnaires, and parents gave passive informed consent for the participation of their child. This trial was approved by the Ethical Committee of the Faculty of Social and Behavioral Sciences of Utrecht University (FETC17-05) and registered in the Dutch Trial Registration, number NL6371 (old number NTR6554; see for protocol Mertens et al., 2018).

\section{Participants}

At baseline, 12997 th grade students with an average age of 12.38 years $(S D=.62)$ participated. About half of the participants $(n=661 ; 54 \%)$ were boys and most $(n=815 ; 69 \%)$ had a Western background. ${ }^{1}$ In the R\&W condition, students $(n=925)$ were on average 12.35 years old $(S D=.61)$ of whom $462(52 \%)$ were boys and 617 (73\%) had a Western background. In the control condition, students $(n=374)$ were on average 12.47 years old $(S D=.64)$ of whom $199(57 \%)$ were boys and 198 (59\%) had a Western background.

Students in the R\&W condition were slightly younger $\left(F(1,1230)=10.46, p=.001, \eta_{\text {partial }}^{2}=.008\right)$, and more had a Western background $\left(\chi^{2}(1)=20.07, p<.001, \varphi=-.130\right)$ than students in the control condition. Age and ethnic background were therefore added as a covariate in the analyses.

\section{Attrition}

In total, 472 (36\%) students completed the questionnaires at all waves and $1170(90 \%)$ completed the questionnaires at least 3 of the 6 waves. Chi-square difference tests and a MANOVA showed that students without missing data did not differ from students with missing data on gen$\operatorname{der}\left(\chi^{2}(1)=1.30, p=.254, \varphi=.032\right)$, age, and outcomes $\left(F(6,1210)=1.57, p=.152, \eta_{\text {partial }}^{2}=.008\right)$. Concerning ethnic background, students without missing data had slightly more often a Western background than students with missing data $\left(\chi^{2}(1)=7.61, p=.006, \varphi=.080\right)$.

\section{Conditions}

\section{$\mathrm{R} \& \mathrm{~W}$}

Students received $14 \mathrm{R} \& \mathrm{~W}$ lessons of the manualized program (Ykema, 2002, 2018). The lessons were implemented weekly during 90-min physical education lessons. Trainers were teachers of the schools, mostly physical education teachers, who completed the 3-day training to become certified R\&W trainers. During the lessons, students

\footnotetext{
1 Ethnic background was coded as Western (i.e., both parents born in Europe, North-America, Oceania, Indonesia, or Japan; Central Bureau for Statistics, 2021) or non-Western (all others).
} 
Fig. 1 Flow chart

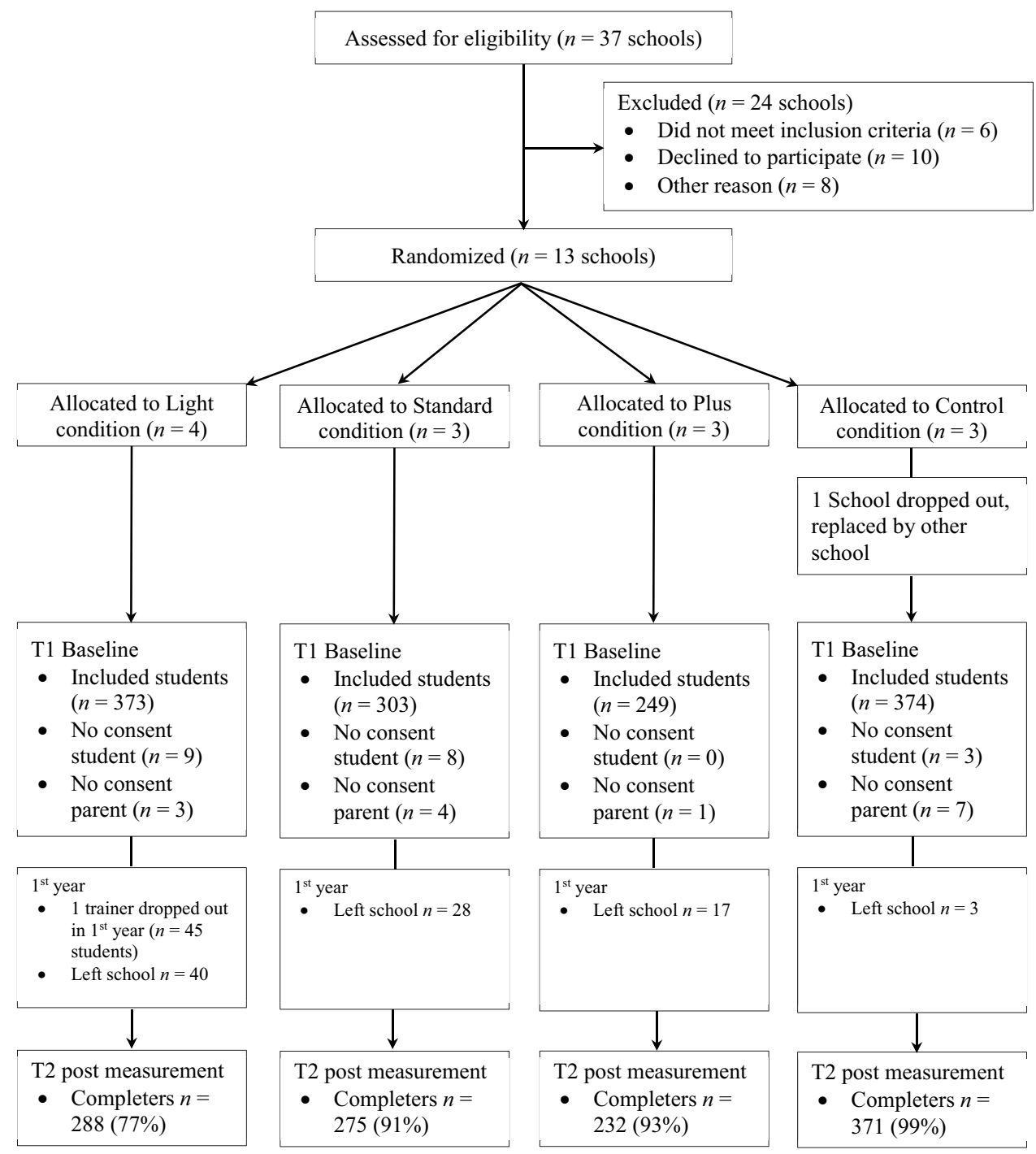

participated in physical games and exercises, reflected on the exercises, shared and discussed their thoughts with each other, and addressed how to use the learned skills in their daily lives.

Communication during the intervention is framed within the symbolic principles of "rock" (i.e., an uncompromising attitude in which one is able to resist pressure from others) and "water" (i.e., a flexible and cooperative attitude in which one is open to opinions, thoughts, and feelings of others) representing opposite ends of a continuum (see the study protocol for more information Mertens et al., 2018).

Treatment fidelity was assessed with self-reports of the R\&W trainers and with observations of $67 \mathrm{R} \& \mathrm{~W}$ lessons by $3 \mathrm{R} \& \mathrm{~W}$ experts. Overall, the majority of the $\mathrm{R} \& \mathrm{~W}$ lessons were indeed implemented and fidelity to the manual was moderate to high (see Mertens et al., 2021 for an elaborate description of treatment fidelity).

\section{Control}

Schools in the control condition implemented care as usual which differed between schools. For instance, students were assigned a teacher as personal coach to discuss their wellbeing. Additionally, schools facilitated actions to prevent or stop bullying such as a project week about "being different," an anti-bullying contract, and discussing bullying in the class or issues were addressed by an "anti-bulling coordinator."

\section{Measurements}

\section{Positive Outcomes}

Resilience The ability to adapt to change and stressful events in a healthy and flexible way was assessed with 3 items (e.g., 
"Able to adapt to change.") of the Connor-Davidson Resilience Scale - short version (Davidson \& Connor, 2017) rated on a 5 -point Likert-type scale $(0=$ not true at all to $4=$ true nearly all the time; Cronbach's $\alpha=.56-.70)$.

Psychological Wellbeing The presence of positive feelings and absence of negative feelings were measured using the subscale Psychological wellbeing of the KIDSCREEN-27 (Ravens-Sieberer \& The European KIDSCREEN Group, 2006) with 2 items (e.g., "Past week, have you been in a good mood?") rated on a 5-point Likert-type scale ( $1=$ never to $5=$ always; Cronbach's $\alpha=.67-.81$ ).

\section{Self-awareness}

Emotional Self-regulation The ability to control emotions and access to emotion regulation strategies was measured using 4 items (e.g., "When I'm upset, I become out of control.") of the Difficulties in Emotion Regulation Scale (Anderson et al., 2016) answered on a 5-point Likert-type scale $(1=$ almost never to $5=$ almost always $)$. All items were recoded so that high scores indicate high levels of emotional self-regulation (Cronbach's $\alpha=.73-.84$ ).

Self-esteem Feelings of global self-worth was assessed with 3 items (e.g., "I am satisfied with myself.") of the subscale global self-perception of the Self-perception Profile (Harter, 1988) answered on a 4-point Likert-type scale ( $1=$ completely not true to $4=$ completely true). Some items were recoded so that high scores indicated high levels of self-esteem (Cronbach's $\alpha=.64-.71)$.

Self-reflection Students completed 3 items (e.g., "I often think about how I feel about something.") of the subscale Engage in reflection of the Self-reflection and Insight Scale (Sauter et al., 2010) to measure the extent to which students inspect and evaluate personal thoughts, feelings, and behaviors. The items were preceded by a definition of self-reflection and answered on a 6-point Likert scale $(1=$ disagree strongly to $6=$ agree strongly; Cronbach's $\alpha=.74-86$ ).

\section{Statistical Analyses}

An intention-to-treat approach was employed, that is, all students who were randomized were included in the analyses regardless of their level of attendance to the assigned condition. In order to include all participants in the models, full information maximum likelihood (FIML) procedures were used with robust maximum likelihood estimation (MLR) to obtain parameter estimates, which is robust to non-normality and non-independence of observations.
Clustering at school level was taken into account using the complex sample cluster feature of Mplus (version 8.2; Muthén \& Muthén, 2017). This conservative clustering procedure provides unbiased estimates of the standard errors (Muthén \& Muthén, 2017). Clustering at class level was not taken into account due to generally low and nonsignificant intraclass correlations at this level (e.g., Cross et al., 2016).

Parallel changes were analyzed by testing a series of parallel process latent growth curve models (LGC; Cheong et al., 2003). First, we modeled unconditional LGC models for each outcome to assess change over time (regardless of condition). LGC models estimate two latent variables that describe the average initial level (i.e., intercept) and average change over time (i.e., slope) based on growth curves of individual participants (Muthén \& Muthén, 2017). Model fit was determined based on the RMSEA (good fit $<.05$ ), CFI (good fit $>.95$ ), and SRMR (good fit $<.08$; Schermelleh-Engel et al., 2003). Second, the LGC model of one dimension of self-awareness was combined with the LGC model of one positive outcome into one parallel process growth curve model by regressing the slope of the positive outcome on the slope of the dimension of self-awareness. Students' age and ethnic background were added as covariates. A significant regression coefficient between the two slopes indicated that the trajectories of change of the constructs were related. Third, a dummy variable representing the intervention condition $(1=$ intervention, $0=$ control) was added to the model by regressing the slopes of the positive outcome and of the dimension of self-awareness on the dummy variable. To control for initial differences between conditions, we also regressed the intercepts of the positive outcome and of the dimension of self-awareness on the dummy variable. Whether the intervention affected the parallel change of the constructs was examined by modeling the indirect path from condition to outcome via the dimension of self-awareness.

\section{Results}

\section{Unconditional Growth Models}

The descriptives of the concepts per measurement point per condition are shown in Table 1. To assess change over time, we modelled unconditional linear growth models for each concept separately. Factor loadings were set at 0 , $2,3.5,5,6.5$, and 7.5 , representing a time span between the measurement points of respectively 4 weeks, 3 weeks, 


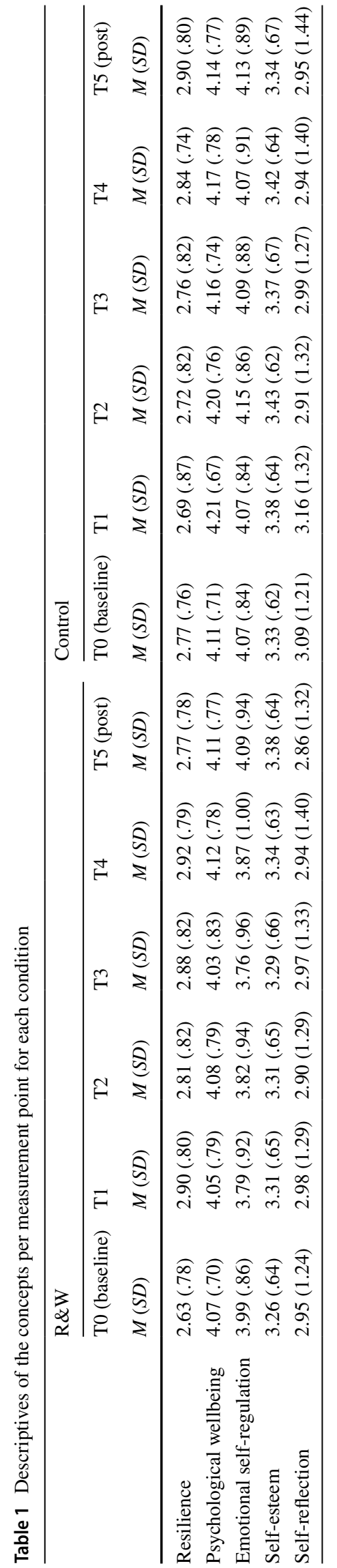

Table 2 Unstandardized estimates of intercepts and slopes and model fit statistics of unconditional linear growth models

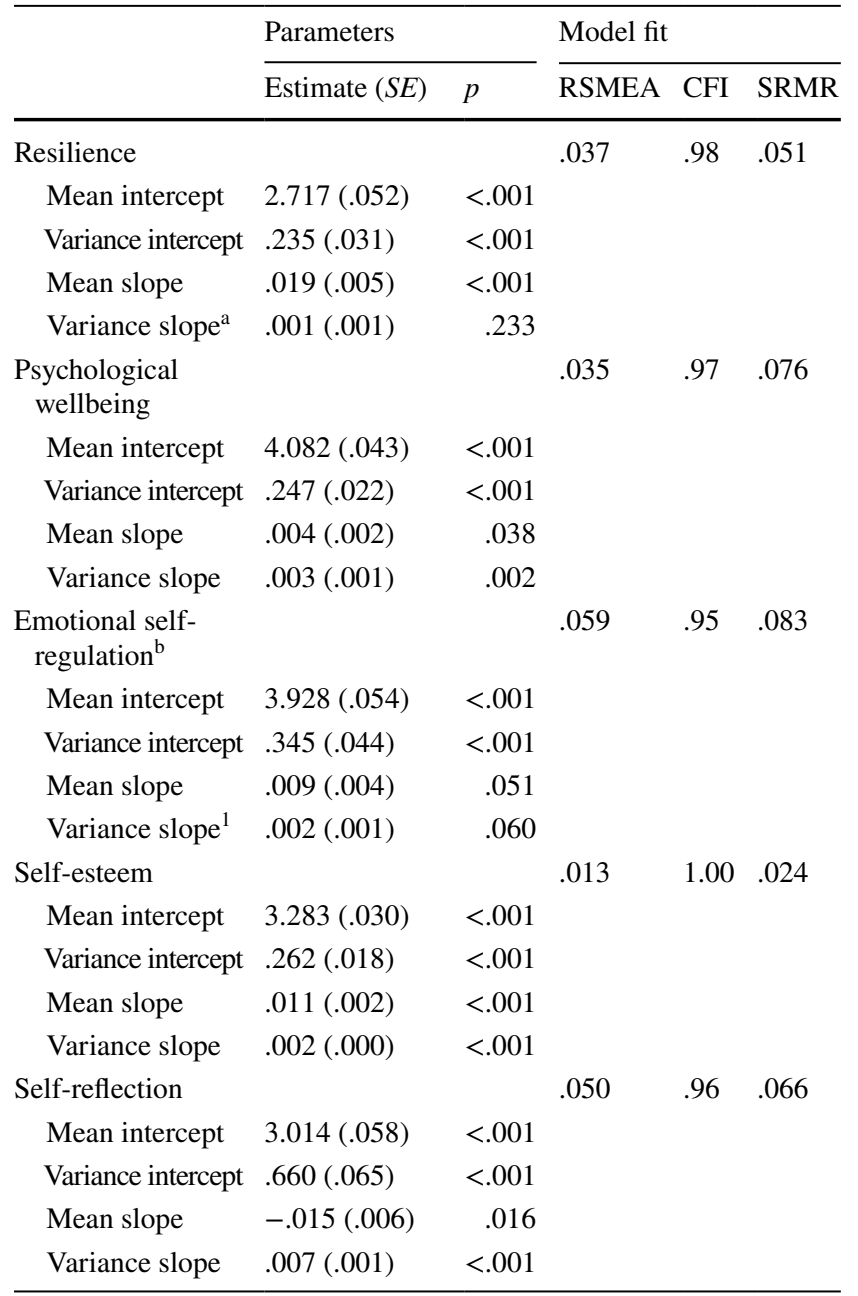

Standardizing estimates is complicated in multilevel models as variances used for standardization are located at the within and between levels (Muthén \& Muthén, 2017). Since it is not our aim to compare multiple predictors within a model, standardized estimates are not necessary. Therefore, we reported the unstandardized variances

${ }^{a}$ Although the slope variance is not significant (or only marginally significant), these variables were included in the analyses, because power to detect slope variance is low due to a too conservative standard chi-square test - the true distribution of variances in LGC models follows a different distribution (Stoel et al., 2006)

${ }^{\mathrm{b}}$ A quadratic LGC model did not significantly improve model fit

3 weeks, 3 weeks, and 2 weeks. All models showed an acceptable to good model fit indicating that the linear growth models adequately described the changes in the concepts. Across all participants, there was a slight increase in resilience, psychological wellbeing, emotional self-regulation $(p=.051)$, and self-esteem. Regarding self-reflection, there was a slight decrease over time (see Table 2 for unstandardized estimates of the intercepts and slopes and the model fit statistics of the linear growth models). 
Table 3 Unstandardized parameter estimates and model fit of the parallel process latent growth models

\begin{tabular}{|c|c|c|c|c|c|}
\hline & \multicolumn{2}{|l|}{ Path b } & \multicolumn{3}{|l|}{ Model fit } \\
\hline & $B(S E)$ & $p$ & RMSEA & CFI & SRMR \\
\hline \multicolumn{6}{|l|}{ Resilience } \\
\hline $\begin{array}{l}\text { Emotional self- } \\
\text { regulation }\end{array}$ & $.38(.17)$ & .022 & .037 & .95 & .047 \\
\hline Self-esteem & $.94(.08)$ & $<.001$ & .023 & .99 & .032 \\
\hline Self-reflection & $-.00(.09)$ & .984 & .030 & .97 & .044 \\
\hline \multicolumn{6}{|c|}{ Psychological wellbeing } \\
\hline $\begin{array}{l}\text { Emotional self- } \\
\text { regulation }\end{array}$ & $.52(.14)$ & $<.001$ & .035 & .96 & .052 \\
\hline Self-esteem & $.91(.15)$ & $<.001$ & .026 & .98 & .045 \\
\hline Self-reflection & $.04(.08)$ & .612 & .030 & .96 & .052 \\
\hline
\end{tabular}

Path b: self-awareness $\rightarrow$ positive outcome

\section{Parallel Change}

All parallel process LGC models showed a good model fit. Increases in emotional self-regulation were related to increases in both resilience and psychological wellbeing. Also, increases in self-esteem were related to increases in resilience as well as psychological wellbeing. Changes in self-reflection, however, were not related to changes in resilience nor to changes in psychological wellbeing (see Table 3).

\section{Parallel Change in Intervention Context}

When the intervention context was taken into account, the parallel changes between the dimensions of self-awareness and the positive outcomes remained the same and were similar in both the intervention condition and the control condition (i.e., care as usual); condition did not predict changes in the three dimensions of self-awareness nor in the two positive outcomes. Additionally, the indirect effects of condition on the positive outcomes through the dimensions of self-awareness were, unsurprisingly, not significant in all models (see Table 4).

\section{Discussion}

In the current study, we examined whether changes in youth's self-awareness were related to concurrent changes in positive development, as suggested in CASEL's conceptual framework (Greenberg et al., 2017). We focused on multiple dimensions of self-awareness (i.e., emotional self-regulation, self-esteem, and self-reflection) and on positive development within an at risk group of early adolescents. In general, the adolescents showed a positive development by slightly improving on emotional self-regulation, self-esteem, resilience, and psychological wellbeing. Concerning self-reflection, however, youth slightly decreased. Increases in two dimensions of self-awareness, i.e., emotional self-regulation and self-esteem, were related to increases in resilience and psychological wellbeing, in line with previous research (e.g., Mann et al., 2004; Morrish et al., 2018). Changes in self-reflection were related neither to changes in resilience nor to changes in psychological wellbeing. These associations between the trajectories of self-awareness and of positive development were the same in an intervention context.

Concerning emotional self-regulation, stimulating youth's competence in regulating their emotions might help them to up-regulate positive emotions and down-regulate negative emotions (Morrish et al., 2018). This ability enables youth to modify and appraise events in order to intensify positive affect or to buffer against negative affect and stressful events (Morrish et al., 2018; Quoidback et al., 2015). Experiencing positive emotions, and few negative emotions, may positively change situations (e.g., people smile at you), could facilitate an easy recollection of positive memories, and might shape the way an event is remembered (Quoidback et al., 2015). In sum, the positive affect together with decreases in negative affect can stimulate youth's resilience and positive wellbeing.
Table 4 Unstandardized parameter estimates of the parallel process latent growth models in intervention context

\begin{tabular}{|c|c|c|c|c|c|c|c|c|}
\hline & \multicolumn{2}{|l|}{ Path c } & \multicolumn{2}{|l|}{ Path a } & \multicolumn{2}{|l|}{ Path b } & \multicolumn{2}{|c|}{ Indirect effect } \\
\hline & $B(S E)$ & $p$ & $B(S E)$ & $p$ & $B(S E)$ & $p$ & $B(S E)$ & $p$ \\
\hline \multicolumn{9}{|l|}{ Resilience } \\
\hline Emotional self-regulation & $.00(.01)$ & .839 & $.00(.01)$ & .821 & $.37(.17)$ & .031 & $.00(.00)$ & .816 \\
\hline Self-esteem & $-.01(.01)$ & .161 & $.01(.01)$ & .200 & $.93(.08)$ & $<.001$ & $.01(.01)$ & .210 \\
\hline Self-reflection & $-.00(.01)$ & .615 & $.01(.01)$ & .111 & $-.00(.08)$ & .988 & $.00(.00)$ & .988 \\
\hline \multicolumn{9}{|l|}{ Psychological wellbeing } \\
\hline Emotional self-regulation & $.00(.01)$ & .838 & $-.00(.01)$ & .834 & $.52(.15)$ & $<.001$ & $-.00(.00)$ & .838 \\
\hline Self-esteem & $-.01(.01)$ & .363 & $.01(.01)$ & .481 & $.91(.16)$ & $<.001$ & $.01(.01)$ & .514 \\
\hline Self-reflection & $.00(.00)$ & .538 & $.02(.01)$ & .090 & $.04(.08)$ & .616 & $.00(.00)$ & .605 \\
\hline
\end{tabular}

Path c: condition $\rightarrow$ positive outcome; path a: condition $\rightarrow$ dimension self-awareness; path b: dimension self-awareness $\rightarrow$ positive outcome 
Regarding self-esteem, high levels of self-esteem can reduce the impact of stressful events and failure (e.g., Blossom et al., 2020; Mann et al., 2004). Confident youth trust their abilities to effectively deal with daily problems (Blossom et al., 2020). They experience daily events as more positive and negative events as less important than youth with low levels of selfesteem, enabling them to reach their full potential and decreases their chances of developing internalizing or externalizing problem behaviors (Mann et al., 2004). In addition, when faced with failure, youth with high levels of self-esteem become extra motivated in pursuing their goals, while youth with low levels of self-esteem experience a further decline in self-esteem and positive affect, generalize the failure to other aspects of their selves, and disengage from their goals as they lose confidence in their abilities (Park et al., 2007). Thus, as for improvements in emotional self-regulation, increases in youth's self-esteem can improve their psychological wellbeing and diminish the effects of negative events which may increase their resilience and ultimately stimulate their overall positive development.

In contrast, changes in self-reflection were not related to concurrent changes in youth's positive development. Perhaps the mere act of reflecting on one's own behavior, thoughts, and feelings is not enough for improvements in one's development since this inspection can be independent of emotion and potential effects that arise due to this inspection (Stein \& Grant, 2014). Also, other studies found no relations between self-reflection and youth's positive development. However, they did find relations between self-insight and positive youth development (e.g., Lyke, 2009; Stein \& Grant, 2014). Hence, rather than solely engage in self-reflection, it may be necessary to also understand one's own behaviors, thoughts, and feelings. This understanding could contribute to one's selfinsight. Self-insight indicates the degree to which individuals understand their behavior, cognitions, and emotions — "ahhah" moments of understanding (Stein \& Grant, 2014). For instance, Lyke (2009) found that engaging in self-reflection was not related to happiness and life satisfaction, but high levels of self-insight were related to higher levels of these outcomes. Additionally, Stein and Grant (2014) found no direct relation between self-reflection and subjective wellbeing, but did find an indirect effect: More self-reflection was related to increases in self-insight which in turn could improve subjective wellbeing. The associations between the trajectories of change of dimensions of self-awareness and of youth's positive development were similar in both the intervention and control group; in both conditions, students improved on emotional self-regulation, self-esteem, resilience, and psychological wellbeing. Although these improvements may represent adolescents' typical development, this finding can also indicate that $\mathrm{R} \& \mathrm{~W}$ and usual care at schools are equally effective which, in turn, suggests that care as usual in Dutch schools is of good quality. Notwithstanding this positive finding, it is important to examine how universal school-based interventions in general, and R\&W specifically, can be optimized as intervention effects of this type of intervention are generally small (e.g., Mertens et al., 2020; Tanner-Smith et al., 2018). One way to optimize R\&W may be by adjusting the dosage of some components. Interventions that address general interpersonal and emotional skills appear more effective when psychoeducation (3-6 exercises) and skill-building exercises (10-20 exercises) are included (De Mooij et al., 2020). During R\&W, these components are implemented, but perhaps not in the right dosage. Thus, future research aiming to optimize interventions should focus on potentially effective components as well as the optimum dosage of these components.

Furthermore, our findings highlight positive developments in youth generally considered as "at risk." Research examining adolescents following the prevocational education track often portrays these adolescents as "problematic students," emphasizing the increased risk of maladjustment, such as behavioral problems, problems with peers, and substance use (e.g., Stevens \& Vollebergh, 2018). However, based on the results of our study, this group of adolescents also shows positive developments on multiple competencies, i.e., emotional self-regulation, self-esteem, resilience, and psychological wellbeing, regardless of whether they received an intervention specially developed to target those outcomes or usual care of schools. In addition, the improvements in the competencies emotional self-regulation and self-esteem support the assumption that metacognitions such as self-awareness develop during early adolescence (Barber, 2005). Perhaps the current view of adolescents following the prevocational education track is more negative then needed due to the focus on their deficits, neglecting their potential strengths (Benson et al., 2007).

When evaluating the significance of the results, it is important to consider some strengths and limitations of the current study. Strengths of the study were the large sample of early adolescents considered as "at risk," examining multiple dimensions of self-awareness, and multiple assessments of the concepts during the intervention. This enabled us to focus on the positive development of "atrisk" youth, to study a phase in which youth appear particularly sensitive for stimulation of their metacognitions, and to examine whether all dimensions of self-awareness have the same association with positive youth development. The multiple measurements enabled us to analyze parallel changes in self-awareness and positive development through latent growth models. A limitation of parallel process LGC models is, however, that the trajectories of change in the concepts are a function of the same period due to which the analysis is not sufficient to prove the direction of causality (Kazdin, 2007). Furthermore, 
conducting multiple assessments during the intervention was only feasible with short questionnaires which limited us in the number of items we could use. As a consequence, items measuring self-reflection were focused on youth's reflection on their thoughts and feelings, but not on their behavior. As reflecting on behavior is part of the concept of self-reflection (Lyke, 2009), lacking an item on this aspect of self-reflection might have affected the precision of the assessment of this concept. In addition, the metacognition to meaningfully reflect on thoughts and feelings is still developing in early adolescents (Barber, 2005) and might be more difficult than reflecting on behaviors. Perhaps this is not the best developmental phase to measure self-reflection; improvements on this competence may emerge later in adolescents' development. Last, we analyzed multiple models without adjusting the $p$-value for multiple tests. Nevertheless, our results showed a consistent pattern across the different models.

The present study makes a valuable contribution to both research and intervention development. First, it seems important to consider parallel development between constructs. Rather than examining whether self-awareness at baseline affected positive outcomes at a later time point, we showed that developments in dimensions of selfawareness, specifically emotional self-regulation and selfesteem, and positive outcomes affect each other in a parallel process meaning that changes in self-awareness are related to concurrent changes in positive youth development. Second, our study showed the importance of focusing on positive development in groups generally conceived as at risk of maladjustment. Although most studies examining prevocational students show their increased risks of developing problems (e.g., Stevens \& De Looze, 2018), our study showed their positive development (regardless of the condition they participated in). Third, youth's positive development can be affected by multiple factors among which the competencies of emotional self-regulation and self-esteem. Based on our findings and in line with CASEL's framework, universal school-based interventions aiming to stimulate positive youth development may potentially be optimized with an additional focus on these two competencies. Examining which specific competencies are important to strengthen in intervention is eminent in order to understand how to optimize interventions and their effectiveness. This knowledge is especially important regarding universal interventions given their generally small effect sizes. To this end, the findings of the present study can be used to build on by future research by studying how emotional self-regulation and self-esteem can best be addressed in an intervention.

Supplementary Information The online version contains supplementary material available at https://doi.org/10.1007/s11121-022-01345-9.
Funding This study was funded by a grant from The Netherlands Organization for Health Research and Development, grant number 531001106

\section{Declarations}

Ethics Approval All procedures in the study involving human participants were in accordance with the ethical standards as laid down in the 1964 Declaration of Helsinki and its later amendments or comparable ethical standards. Ethical approval for this study was obtained by the Ethical Committee of the Faculty of Social and Behavioral Sciences of Utrecht.

Consent to Participate Informed consent was obtained from all individual participants included in the study and students' legal guardians.

Conflict of Interest The authors declare that they have no conflict of interest.

Open Access This article is licensed under a Creative Commons Attribution 4.0 International License, which permits use, sharing, adaptation, distribution and reproduction in any medium or format, as long as you give appropriate credit to the original author(s) and the source, provide a link to the Creative Commons licence, and indicate if changes were made. The images or other third party material in this article are included in the article's Creative Commons licence, unless indicated otherwise in a credit line to the material. If material is not included in the article's Creative Commons licence and your intended use is not permitted by statutory regulation or exceeds the permitted use, you will need to obtain permission directly from the copyright holder. To view a copy of this licence, visit http://creativecommons.org/licenses/by/4.0/.

\section{References}

Anderson, L. M., Reilly, E. E., Gorrell, S., Schaumberg, K., \& Anderson, D. A. (2016). Gender-based differential item function for the difficulties in emotion regulation scale. Personality and Individual Differences, 92, 87-91. https://doi.org/10.1016/j.paid.2015.12.016

Bakker, D., \& Rickard, N. (2018). Engagement in mobile phone app for self-monitoring of emotional wellbeing predicts changes in mental health: MoodPrism. Journal of Affective Disorders, 227, 432-442. https://doi.org/10.1016/j.jad.2017.11.016

Barber, B. K. (2005). Positive interpersonal and intrapersonal functioning: An assessment of measures among adolescents. In K. A. Moore, \& L. H. Lippman (Eds.), What do children need to flourish: Conceptualizing and measuring indicators of positive development (pp. 147-161). New York, NY, US: Springer Science and Business Media.

Benson, P. L., Scales, P. C., Hamilton, S. F., \& Sesma, A. Jr. (2007). Positive youth development: Theory, research, and applications. In R. M. Lerner (Ed.), Handbook of child psychology. Volume 1: Theoretical models of human development ( $6^{\text {th }}$ ed., pp. 894-941). John Wiley \& Sons. https://doi.org/10.1002/9780470147658. chpsy0116

Blossom, J. B., Adrian, M. C., Vander Stoep, A., \& McCauley, E. (2020). Mechanisms of change in the prevention of depression: An indicated school-based prevention trial at the transition to high school. Journal of American Academy of Child and Adolescent Psychiatry, 59, 541-551. https://doi.org/10.1016/j.jaac.2019.05. 031

Catalano, R. F., Berglund, M. L., Ryan, J. A. M., Lonczak, H. S., \& Hawkins, J. D. (2004). Positive youth development in the United 
States: Research findings on evaluations of positive youth development programs. The Annals of the American Academy, 591, 98-124. https://doi.org/10.1177/0002716203260102

Central Bureau for Statistics. (2021). Wat is het verschil tussen een westerse en niet-westerse allochtoon? [What is the difference between a Western and non-Western background?]. https://www. cbs.nl/nl-nl/faq/specifiek/wat-is-het-verschil-tussen-een-westerseen-niet-westerse-allochtoon. Accessed 11 Jun 2021.

Cheong, J., MacKinnon, D. P., \& Toon Khoo, S. (2003). Investigation of mediational processes using parallel process latent growth curve modeling. Structural Equation Modeling: A Multidisciplinary Journal, 10, 238-262. https://doi.org/10.1207/ S15328007SEM1002_5

Cross, D., Shaw, T., Hadwen, K., Cardoso, P., Slee, P., Roberts, C., Thomas, L., \& Barnes, A. (2016). Longitudinal impact of the Cyber Friendly Schools program on adolescents cyberbullying behavior. Aggressive Behavior, 42, 166-180. https://doi.org/10.1002/ab.21609

Davidson, J. R. T., \& Connor, K. M. (2017). Connor-Davidson Resilience Scale (CD-RISC) manual. Unpublished and partly accessible at https://www.cd-risc.com

De Graaf, I., De Haas, S., Zaagsma, M., \& Wijssen, C. (2016). Effects of rock and water: An intervention to prevent sexual aggression. Journal of Sexual Aggression, 22, 4-19. https://doi. org/10.1080/13552600.2015.1023375

De Mooij, B., Fekkes, M., Scholte, R. H. J., \& Overbeek, G. (2020). Effective components of social skills training programs for children and adolescents in nonclinical samples: A multilevel meta-analysis. Clinical Child and Family Psychology Review, 23, 250-264. https://doi.org/10.1007/s10567-019-00308-x

Elliott, I., \& Coker, S. (2008). Independent self-construal, self-reflection, and self-rumination: A path model for predicting happiness. Australian Journal of Psychology, 60, 127-134. https://doi.org/10.1080/ 00049530701447368

Greenberg, M. T., Domitrovich, C. E., Weissberg, R. P., \& Durlak, J. A. (2017). Social and emotional learning as a public health approach to education. The Future of Children, 27(1), 13-32. https://www.jstor.org/stable/44219019

Harter, S. (1988). Manual for the self-perception profile for adolescents. Denver, US: university of Denver

Kazdin, A. E. (2007). Mediators and mechanisms of change in psychotherapy research. Annual Review of Clinical Psychology, 3, 1-27. https://doi.org/10.1146/annurev.clinpsy.3.022806.091432

Lyke, J. A. (2009). Insight, but not self-reflection, is related to subjective well-being. Personality and Individual Differences, 46, 66-70. https://doi.org/10.1016/j.paid.2008.09.010

Mann, M., Hosman, C. M. H., Schaalma, H. P., \& De Vries, N. K. (2004). Self-esteem in a broad-spectrum approach for mental health promotion. Health Education Research, 19, 357-372. https://doi.org/10.1093/her/cyg041

Mertens, E. C. A., Deković, M., Leijten, P., Londen, V., \& Reitz, E. (2020). Components of school-based interventions stimulating students' intrapersonal and interpersonal domains: A metaanalysis. Clinical Child and Family Psychology Review, 23, 605-631. https://doi.org/10.1007/s10567-020-00328-y

Mertens, E. C. A., Deković, M., Van Londen, M., \& Reitz, E. (2018). The effectiveness of Rock and Water in improving students' socio-emotional adjustment and social safety: Study protocol for a randomized controlled trial. BMC Psychology, 6, 1-12. https://doi.org/10.1186/s40359-018-0247-y

Mertens, E. C. A., Deković, M., Van Londen, M., \& Reitz, E. (2021). Solid as a rock, flexible as water? Effectiveness of a universal school-based intervention addressing students intrapersonal and interpersonal domain [Manuscript submitted for publication]. Utrecht University.

Morrish, L., Rickard, N., Chyuan Chin, T., \& Vella-Brodrick, D. A. (2018). Emotion regulation in adolescent well-being and positive education. Journal of Happiness Studies, 19, 15431564. https://doi.org/10.1007/s10902-017-9881-y

Muthén, L. K., \& Muthén, B. O. (2017). Mplus user's guide. 8th Edition. Los Angeles, CA: Muthén \& Muthén.

O'Rourke, H. P., \& MacKinnon, D. P. (2018). Reasons for testing mediation in the absence of an intervention effect: A research imperative in prevention and intervention research. Journal of Studies on Alcohol and Drugs, 79(2), 171-181. https://doi.org/ 10.15288/jsad.2018.79.171

Park, L. E., Crocker, J., \& Kiefer, A. K. (2007). Contingencies of self-worth, academic failure, and goal pursuit. Personality and Social Psychology Bulletin, 33, 1503-1517. https://doi.org/10. 1177/0146167207305538

Quoidback, J., Mikolajczak, M., \& Gross, J. J. (2015). Positive interventions: An emotion regulation perspective. Psychological Bulletin, 141, 655-693. https://doi.org/10.1037/a0038648

Ravens-Sieberer, U., \& The European KIDSCREEN Group. (2006). The KIDSCCREEN questionnaires. Quality of life questionnaires for children and adolescents - Handbook. Pabst Science publisher.

Rimm-Kaufman, S. E., \& Hulleman, C. S. (2014). Social and emotional learning in elementary school settings: Identifying mechanisms that matter. In J. Durlak \& R. Weissberg (Eds.), The handbook of social and emotional learning (pp. 151-166). Guilford.

Sauter, F. M., Heyne, D., Blöte, A. W., Van Widenfelt, B. M., \& Westenberg, P. M. (2010). Assessing therapy-relevant cognitive capacities in young people: Development and psychometric evaluation of the self-reflection and insight scale for youth. Behavioral and Cognitive Psychotherapy, 38, 303-317. https:// doi.org/10.1017/S1352465810000020

Schermelleh-Engel, K., Moosbruger, H., \& Müller, H. (2003). Evaluating the fit of structural equation models: Tests of significance and descriptive goodness-of-fit measures. Methods of Psychological Research Online, 8, 23-74.

Stein, D., \& Grant, A. M. (2014). Disentangling the relationships among self-refleciton, insight, and subjective well-being: The role of dysfunctional attitudes and core self-evaluations. The Journal of Psychology, 148, 505-522. https://doi.org/10.1080/ 00223980.2013 .810128

Stevens, G., \& De Looze, M. (2018). Welbevinden en psychische problemen [Wellbeing and psychological problems]. In M. Boer \& G. Stevens (Eds.), HBSC 2017: Gezondheid, welzijn en opvoeding van jongeren in Nederland [Health, wellbeing, and child rearing of youth in the Netherlands] (pp. 63-75). Ridderprint BV.

Stevens, G., \& Vollebergh, W. (2018). Nabeschouwing [Commentary]. In M. Boer \& G. Stevens (Eds.), HBSC 2017: Gezondheid, welzijn en opvoeding van jongeren in Nederland [Health, wellbeing, and child rearing of youth in the Netherlands] (pp. 165-181). Ridderprint BV.

Stoel, R. D., Garre, F. G., Dolan, C., \& Van den Wittenboer, G. (2006). On the likelihood ratio test in structural equation modeling when parameters are subject to boundary constraints. Psychological Methods, 11, 439-455.

Sutton, A. (2016). Measuring the effects of self-awareness: Construction of the self-awareness outcomes questionnaire. Europe's Journal of Psychology, 12, 645-658. https://doi.org/10.5964/ ejop.v12i4.1178

Tanner-Smith, E. E., Durlak, J. A., \& Marx, R. A. (2018). Empirically based mean effect size distributions for universal prevention programs targeting school-aged youth: A review of metaanalyses. Prevention Science, 19, 1091-1101. https://doi.org/ 10.1007/s11121-018-0942-1

Weytens, F., Luminet, O., Verhofstadt, L. L., \& Mikolajczak, M. (2014). An integrative theory-driven positive emotion regulation intervention. PLoS One, 9(4), article e95677. https://doi. org/10.1371/journal.pone.0095677 
Ykema F. (2002). Het Rots en Water perspectief. Een psychofysieke training voor jongens. Basisboek [The Rock and Water perspective. A psychophysical training for boys. Basic book]. Uitgeverij SWP.

Ykema F. (2018). Rots en Water praktijkboek. Psychofysieke training voor jongens én meisjes [Rock and Water practice book. Psychophysical training for boys and girls]. Uitgeverij SWP.

Ykema F., Hartman, D., \& Imms, W. (2006). Bringing it together. Includes 22 case studies of Rock \& Water in practice in various settings. University of Newcastle, Family action Centre and Gadaku institute.

Publisher's Note Springer Nature remains neutral with regard to jurisdictional claims in published maps and institutional affiliations. 